\title{
Research Developments of Group Decision and Negotiation in Latin America
}

\author{
Adiel Teixeira de Almeida • \\ Ana Paula Cabral Seixas Costa
}

Published online: 1 December 2011

(C) Springer Science+Business Media B.V. 2011

\section{Introduction}

Group decision and negotiation (GDN) are present in any organization, particularly at the top management level of public and private organizations. Applications of GDN models may be found in a variety of situations, such as when undertaking strategic planning, and taking decisions on investments and on public policy in several issues, for instance in water management.

Developments in GDN for Latin America (LA) include a diversity of practical application contexts and methodological topics marching the extent to which cultural issues are varied in LA. GDN processes have broadened the scope of GDN due to several cultural aspects. These stem from practical applications which have given insights into dealing with new methodological issues. The context in which these applications are conducted may have an important influence on procedures for aggregating group preferences, including the theoretical and methodological issues addressed.

Some GDN studies in LA are related to the aggregation of experts' knowledge, while others are associated with DMs' preferences. That is, GDN processes may be related to aggregation or integration of either decision makers (DMs) or experts, which may represent different situations. While the aggregation of DMs generally is related to preferences on consequences (Leyva-Lopez and Fernandez-Gonzalez 2003; Espin et al. 2007; Zamora and Burguete 2008; Morais and de Almeida 2012), the aggregation of experts is associated with knowledge of a subject, generally focused on seeking consensus regarding the perception of that particular subject (Medina and

A. T. de Almeida $(\varangle)$. A. P. C. S. Costa

Federal University of Pernambuco, Recife, PE, Brazil

e-mail: adiel.almeida@pq.cnpq.br; almeidaatd@gmail.com

A. P. C. S. Costa

e-mail: apcabral@hotmail.com 
Moreno 2007; Ekel et al. 2008; Parreiras et al. 2010). On the other hand, some models are built in order to tackle mutually these two issues, since both are present in many GDN problems.

A number of studies demonstrate the contributions made by GDN approaches in several different cultural contexts. However, the LA context is one in which neither applied research nor the methods developed have yet been made widely known. When seeking information on GDN research in LA, within the ISI database (October 2011), the results showed the opportunity for new developments and applications in the region. With regard to the topic of "group decision", 25 papers out of 4,038 were found, including from the following Countries/Territories: Argentina, Brazil, Chile, Colombia, Mexico and Venezuela. As to a search on the topic of "negotiation", 347 papers out of 16,067 were found. These were from the following Countries/Territories: Argentina, Barbados, Brazil, Chile, Colombia, Costa Rica, Jamaica, Mexico, Panama, Uruguay and Venezuela. However, the observation needs to be made that most of the studies found under the topic of "group decision" are related to GDN, while many studies found under the topic of "negotiation" are not actually related to GDN.

Many studies of GDN in LA deal with water research (Morais and de Almeida 2007, 2010; Wester et al. 2008; Munoz-Pina et al. 2008; Silva et al. 2010). Other studies deal with a broad range of contexts, such as: Scheduling meetings (Wainer et al. 2007), Business Process Management (Romero and Molina 2009) and accidents arising from oil spills at sea (Krohling and Campanharo 2011). One of the studies compares cultural issues in the context of GDN (Metcalf et al. 2006). While research comparing cultures issues in GDN is scarce, this study presents a comparative analysis. It compares Mexico and countries in other regions, representing five cultural clusters, and identifies ten ways on how culture affects negotiating style. The study confirms there are significant differences in GDN orientations between different cultures.

\section{Special Issue on GDN in LA}

This special issue aims to gather research which expresses methodological contributions and practical applications from LA. The articles cover many situations, such as strategic planning and public policy decision making. Some papers offer insights from practice in the LA context and all of them deal with theoretical and methodological developments for GDN.

The first paper deals with a model called a Compensatory Negotiation Solution by Knowledge Engineering (CNSKE). This model puts forward a proposal for combining experts' knowledge of experts in a negotiation process. In this model the experts' propositions are given in a natural language, so their knowledge is represented by logic predicates. Then, calculations are made based on operators of compensatory fuzzy logic, which satisfy the axioms of utility. Coalition possibilities are evaluated and each player can select the best coalition in the negotiation process. The proposed model is applied to an example of a bargaining problem, in which three cement companies are trying to reach an agreement on how to avoid harmful competition.

In the subsequent paper, three consensus models deal with the aggregation of multiple experts' opinions for a multicriteria group decision making problem. These models are based on a fuzzy approach and each one has a different scheme for aggregating 
expert's opinions in order to build a collective result. These three approaches are: the aggregation of individual evaluations; the aggregation of individual preferences per criterion, and; the aggregation of individual results. The paper discusses the basic conditions to be considered when selecting one of these aggregation schemes to achieve consensus. An analysis is made of how adequate they are for a specific real-world problem. The expert members of the group are integrated into a cooperative work and each consensus model allows them to take advantage of their respective capabilities. The model is applied to a strategic planning problem in Brazil.

Next an additive model is presented on aggregating group decisions using the veto concept in the context of a water utility problem. The veto concept is used to build a model in order to overcome some undesirable effects of the compensatory condition of the additive model in the process of aggregating all DMs' preferences. That is, in the additive model, the final recommendation may produce some extremely undesirable solution for one or more DMs. The proposed model can avoid conflicts and regrets that have arisen from this circumstance. This model uses the concept of ranking veto by means of a reduction factor in order to punish conflicting alternatives. This reduces possible disagreements in an additive group decision aggregation model.

In the final paper, planning the use of water resources is tackled using a multi-expert decision-making model. A linguistic-label aggregation based on fuzzy opinion method is applied to environmental and water resources planning in a river basin in Colombia. The study considers temporal linguistic labels and a fuzzy ranking procedure, and can therefore include the fuzzy membership function and the frequency of experts' opinions (the mean and the standard deviation) for each alternative. A computational tool based on the proposed model is developed and presented in this paper. The model contributes to the identification of consensus in the decision process, thus supporting the achievement of long-term economic and ecological sustainability.

All papers cope with problems in the LA context and deal mainly with public policy decisions, particularly in water resources planning. Also the strategic planning of a private company is analyzed. Most papers have an emphasis on aggregating experts' knowledge, using fuzzy models, while, at the same time, all of them are concerned with the aggregation of DMs' preferences.

Acknowledgments We thank the authors for their contributions to this special issue. We are also very grateful to the reviewers for their critiques and valuable suggestions on improving all papers, especially those reviewers who were already more than fully engaged on their activities as Associate Editors. Finally, we would like to express our gratitude to Melvin Shakun, for inviting and supporting us to bring this special issue to completion. His guidance was always stimulating. Mel has systematically provided occasions for connecting the GDN community, thus confirming his inspirational leadership. This special issue confirms his purpose of improving these connections and aggregating Latin American research within the global GDN community.

\section{References}

Ekel P, Queiroz J, Parreiras R, Palhares R (2008) Fuzzy set based models and methods of multicriteria group decision making. Nonlinear Anal Theory Methods Appl 71(12):409-419

Espin R, Fernandez E, Mazcorro G, Lecich MI (2007) A fuzzy approach to cooperative n-person games. Eur J Oper Res 176(3):1735-1751

Krohling RA, Campanharo VC (2011) Fuzzy TOPSIS for group decision making: a case study for accidents with oil spill in the sea. Expert Syst Appl 38(4):4190-4197 
Leyva-Lopez JC, Fernandez-Gonzalez E (2003) A new method for group decision support based on ELECTRE III methodology. Eur J Oper Res 148(1):14-27

Medina S, Moreno J (2007) Risk evaluation in Colombian electricity market using fuzzy logic. Energy Econ 29(5):999-1009

Metcalf LE, Bird A, Shankarmahesh M, Aycan Z, Larimo J, Valdelamar DD (2006) Cultural tendencies in negotiation: a comparison of Finland, India, Mexico, Turkey, and the United States. J World Bus 41(4):382-394

Morais DC, de Almeida AT (2007) Group decision-making for leakage management strategy of water distribution metwork. Resour Conserv Recycl 52(2):441-459

Morais DC, de Almeida AT (2010) Water network rehabilitation: a group decision-making approach. Water SA 36(4):487-794

Morais DC, de Almeida AT (2012) Group decision making on water resources based on analysis of individual rankings. Omega 40:42-45. doi:10.1016/j.omega.2011.03.005

Munoz-Pina C, Guevara A, Torres JM, Brana J (2008) Paying for the hydrological services of Mexico's forests: analysis, negotiations and results. Ecol Econ 65(4):725-736

Parreiras RO, Ekel PY, Martini JSC, Palhares RM (2010) A flexible consensus scheme for multicriteria group decision making under linguistic assessments. Inf Sci 180(7):1075-1089

Romero D, Molina A (2009) VO breeding environments \& virtual organizations integral business process management framework. Inf Syst Frontiers 11(5):569-597

Silva VBS, Morais DC, de Almeida AT (2010) A multicriteria group decision model to support watershed committees in Brazil. Water Resour Manag 24:4075-4091

Wainer J, Ferreira PR Jr, Constantino ER (2007) Scheduling meetings through multi-agent negotiations. Decis Support Syst 44(1):285-297

Wester P, Vargas-Velazquez S, Mollard E, Silva-Ochoa P (2008) Negotiating surface water allocations to achieve a soft landing in the closed Lerma-Chapala basin, Mexico. Int J Water Resour Dev 24(2): 275-288

Zamora LP, Burguete STG (2008) Second-order preferences in group decision making. Open Res Lett 36(1):99-102 